\title{
Plan de las Mujeres: marco conceptual y metodología para el Buen Vivir $^{1}$
}

\author{
Julieta Paredes \\ Mujeres Creando Comunidad \\ Asamblea Feminista
}

\section{Abstract}

This article analyzes the impact that neoliberal policies have on women and sets out the epistemological fracture that communitarian feminism produces in Western feminism. We discuss the circumstances in which, for the first time in the history of Bolivian public policies for women, a Plan de las Mujeres emerges from within women's social organizations. This article also offers the conceptual frame that guides such a Plan, which relies on five categories or fields of direct action that help us to defend ourselves from a market that has put our very lives on sale. These categories are our bodies, our space, our time, our memory, and the movements that we are able to articulate.

Keywords

Chacha-Warmi, Communitarian State, Communitarian Feminism, Deneoliberalization, Depatriarchalizing Principle, Pachamama, Plan de las Mujeres, Plurinational State, Public Policy, Vivir Bien

\footnotetext{
${ }^{1}$ Este trabajo es una versión revisada de las tesis presentadas en Hilando fino. Desde el feminismo comunitario (La Paz: CEDEC, 2008). En su forma actual, fue presentado performativamente en la Universidad de Pittsburgh en Marzo 2009.
} 


\section{Resumen}

Este trabajo analiza el impacto de las políticas neoliberales en la vida de las mujeres y expone el rompimiento epistemológico que el feminismo comunitario produce en el feminismo occidental. Se discuten las circunstancias en las que, por primera vez en la historia de las políticas públicas para las mujeres en Bolivia, surge un Plan desde la base y las experiencias de las organizaciones sociales de mujeres. El trabajo presenta el marco conceptual que orienta este Plan de las Mujeres y que descansa en cinco categorías o campos de acción directa que nos ayudan a defendernos de un mercado que puso en venta nuestras propias vidas. Estas categorías son: nuestros cuerpos, nuestro espacio, nuestro tiempo, nuestra memoria y los movimientos que articulamos.

Palabras claves

Chacha-Warmi, Des-neoliberalización, Estado Comunitario, Estado Plurinacional, Feminismo Comunitario, Pachamama, Plan de las Mujeres, Principio de Despatriarcalización, Políticas Públicas, Vivir Bien

\section{¿Qué es un Plan Quinquenal de las mujeres?}

Cada cinco años los gobiernos hacen un Plan de Políticas Públicas que busca mejorar la situación de las mujeres. Se han hecho cuatro planes quinquenales desde los 80 , pero como estábamos en medio del neoliberalismo, estos planes fueron absorbidos desde el propio sistema y, al final, sirvieron de colchón para sus políticas neoliberales. Hoy, por primera vez en la historia de las políticas públicas para las mujeres en Bolivia, un Plan nace desde la base y las experiencias de las organizaciones sociales de mujeres. Esto se lo debemos a nuestro pueblo, del cual las mujeres somos la mitad, que abrió con sus luchas estos procesos de cambio. Nuestro Plan no nace de las ONGs de mujeres, 
nace de las mujeres luchadoras del pueblo, quienes vamos a hacer respetar el proceso. Es un Plan que se pone a disposición de todas las organizaciones sociales de mujeres para que se reapropien de algo que es ya de todas nosotras.

\section{Es el Plan de todas las mujeres para que el Vivir Bien sea una realidad desde nuestros cuerpos, nuestras luchas y nuestros sueños}

Estamos en un proceso que fue abierto por la lucha y el sacrificio de las organizaciones sociales el año 2003, en las cuales las mujeres alteñas fueron la mayoría. El resultado de este proceso es que hoy tenemos dos modelos de sociedad que se enfrentan, uno es el modelo neoliberal que no quiere soltar el privilegio de que unos pocos vivan a costa de la mayoría. El otro es el modelo de Estado Comunitario que se quiere poner al servicio de la gente que trabaja y trabaja, pero hasta ahora sigue empobrecida.

\section{A continuación, algunos de los aspectos de ambos modelos}

\begin{tabular}{|l|l|}
\hline \multicolumn{1}{|c|}{ Neoliberalismo } & \multicolumn{1}{|c|}{ Nuestro Modelo } \\
\hline $\begin{array}{l}\text { Colonialismo interno, } \\
\text { colonialismo neoliberal, } \\
\text { etnocentrismo blanco }\end{array}$ & $\begin{array}{l}\text { Descolonizaremos la sociedad con la } \\
\text { complementariedad, la reciprocidad } \\
\text { y la solidaridad entre culturas y } \\
\text { cosmovisiones }\end{array}$ \\
\hline $\begin{array}{l}\text { Estado al servicio de intereses } \\
\text { oligarcas y transnacionales }\end{array}$ & $\begin{array}{l}\text { El Estado social plurinacional y } \\
\text { comunitario trabaja para la mayoría } \\
\text { del pueblo, en especial para los y las } \\
\text { más excluidas y empobrecidas }\end{array}$ \\
\hline $\begin{array}{l}\text { Bolivia ya no se desarrolla en su } \\
\text { beneficio, pues lo que } \\
\text { producimos va en beneficio del } \\
\text { mercado mundial }\end{array}$ & $\begin{array}{l}\text { Bolivia soberanamente decide y se } \\
\text { desarrolla para que todas y todos } \\
\text { puedan Vivir Bien }\end{array}$ \\
\hline
\end{tabular}


REVISTA DE ESTUDIOS BOLIVIANOS

\begin{tabular}{|l|l|}
\hline $\begin{array}{l}\text { Democracia pactada entre } \\
\text { partidos que excluyen y } \\
\text { desconocen el voto popular }\end{array}$ & $\begin{array}{l}\text { Construcción del Poder Social a } \\
\text { partir de la presencia efectiva de las } \\
\text { excluidas y excluidos en las } \\
\text { instancias del Poder Político }\end{array}$ \\
\hline $\begin{array}{l}\text { Las mujeres sirven de colchón } \\
\text { para parchear la pérdida de } \\
\text { derechos y beneficios sociales del } \\
\text { pueblo }\end{array}$ & $\begin{array}{l}\text { Las mujeres somos activa y } \\
\text { efectivamente la mitad de todo }\end{array}$ \\
\hline
\end{tabular}

- El colonialismo interno ha servido de base racista para la implementación de políticas de ajuste neoliberal

El modelo neoliberal es parte de la cadena de modelos que buscan explotar a la mayoría para que se privilegien unos pocos (en Bolivia las indígenas somos la mayoría). Como en la colonia, el neoliberalismo desprecia lo indígena, la diferencia es que ya no están los españoles y se ha creado una oligarquía. En el neoliberalismo, aunque seas indígena, quieres blanquearte. Estos nuevos colonizadores, que desde la República han construido sus estructuras de privilegios, son los que se convirtieron en los modernizadores del Estado desde 1985. Son los que querían seguir creyendo que lo indígena estaba recluido a las áreas rurales y no querían ver que estamos en todo lado, también en las ciudades, haciendo Bolivia.

\section{Reducción del Estado a función de árbitro parcializado por los intereses transnacionales \\ El Estado se desentiende de todos los deberes de seguridad social, como la salud, la educación, la vivienda, la jubilación, la inamovilidad laboral, conseguidos por las luchas y conquistas sociales del pueblo trabajador. En los hechos, esto ha significado que las mujeres han sido las encargadas de cubrir estas necesidades sociales desde las familias y comunidades rurales.}

La iniciativa económica y productiva del país, o lo que se entiende por desarrollo, dejó de ser un asunto de Estado y de decisiones de gobierno

Todo queda sometido a las necesidades del mercado, manejado en beneficio de las transnacionales desde centros de poder mundial.

Para implantar esta enajenación de las decisiones de un país han necesitado construir un engaño de democracia 


\section{REVISTA DE ESTUDIOS BOLIVIANOS}

Este engaño es la democracia pactada entre partidos, en la que se legitimaron la mediación y la intermediación de la voluntad popular a través de acuerdos y componendas que hacen los partidos gobernantes y alianzas que se pasan el poder por turno, durante dieciocho años. Por supuesto que los sectores populares y movimientos sociales son también responsables de apoyar con su voto a estos partidos neoliberales.

Descolonizaremos la sociedad con la complementariedad, la reciprocidad y la solidaridad entre culturas, cosmovisiones y formas de ser

Tenemos que dejar de ver a los blancos y blancas como superiores y empezar a considerarlos iguales. Es imprescindible mirar a las hermanas y hermanos de otras culturas y pueblos originarios con respeto y cariño. Entre campo y ciudad no puede haber discriminación; las ciudades nos alimentan con otras vertientes culturales y diferentes maneras de ser. El campo nos nutre con los frutos de la Pachamama y las formas de organizarse que vienen de nuestras abuelas y abuelos.

\section{El estado social plurinacional y comunitario trabaja para la mayoría del pueblo boliviano, especialmente para los y las más excluidas y empobrecidas}

Es claro que los y las empobrecidas deben ser la prioridad para las políticas públicas, y las mujeres somos las más excluidas. A las mujeres se nos tiene que dar dos, hasta tres veces más, si eres mujer, indígena y pobre.

\section{Bolivia soberana y digna decide y se desarrolla para que todas y todos puedan Vivir Bien}

No se puede Vivir Bien si los o las demás viven mal. Se trata de vivir como parte de la comunidad, con protección de ella y no con violencia en ella. Supone esto que todas y todos tengamos salud, casa, comida, escuela. También significa "vivir bien contigo y conmigo", que es diferente del "vivir mejor" occidental, que es individual, separado de los y las demás e inclusive a expensas de los y las demás. De este modo, el Vivir Bien es el acceso y disfrute de todos y todas a bienes materiales y recursos naturales, el disfrute de los productos sociales, artísticos, y la realización en aspectos de la vida como el placer, la afectividad, el desarrollo intelectual y espiritual, la creatividad. Todo ello en armonía con el medio ambiente. 


\section{Construcción del poder social a partir de la presencia efectiva de los excluidos y excluidas en las instancias del poder político, para que la democracia sea de verdad participativa e incluyente}

Hay que superar el Estado burgués con la acción directa, social y política de los pueblos, movimientos y organizaciones sociales y de la sociedad civil, como lo hicimos en octubre 2003. Esto es posible en espacios públicos que consolidan una visión nacional acorde con intereses colectivos y no egoístas, sectoriales o corporativos. Así, las demandas sectoriales y territoriales asumen una visión política nacional y privilegian el bien común mediante asambleas y consejos.

Pero una cosa es lo que queremos, otra es la realidad que vivimos y que nos toca cada día. Por eso analizaremos especialmente la situación de las mujeres

\begin{tabular}{|l|l|}
\hline \multicolumn{1}{|c|}{ Patriarcado Neoliberal } & \multicolumn{1}{|c|}{ Patriarcado Popular } \\
\hline $\begin{array}{l}\text { Una élite de mujeres, legitimada } \\
\text { por el neo-liberalismo, usurpa la } \\
\text { representación de la colectividad } \\
\text { femenina de sectores populares y } \\
\begin{array}{l}\text { actúa a nombre de ésta a través } \\
\text { de partidos políticos y ONGs }\end{array}\end{array}$ & $\begin{array}{l}\text { Aunque en los sectores populares } \\
\text { las mujeres tienen participación, la } \\
\text { misma está subordinada a la } \\
\text { legitimación de la población } \\
\text { masculina, que no quiere } \\
\text { reconocer que las mujeres somos la } \\
\text { mitad de cada pueblo }\end{array}$ \\
\hline $\begin{array}{l}\text { Las mujeres, ya incorporadas } \\
\text { desde la colonia a la matriz } \\
\text { productiva del país, en el } \\
\text { neoliberalismo CUBREN el hambre } \\
\text { y la seguridad social que el Estado } \\
\text { deja sin cumplimiento. Además, } \\
\text { son convocadas a trabajar como } \\
\text { mano de obra barata sin gozo de }\end{array}$ & $\begin{array}{l}\text { Este patriarcado naturaliza la } \\
\text { explotación de las mujeres } \\
\text { estableciendo que su rol es el } \\
\text { trabajo doméstico. No reconoce (o } \\
\text { no quiere reconocer) que las } \\
\text { mujeres realizan constantemente } \\
\text { actividades económicas fuera del } \\
\text { hogar para aportar y fortalecer la }\end{array}$ \\
\hline
\end{tabular}


No queremos ninguno de los dos patriarcados Queremos una comunidad sin explotación de las mujeres

\section{El género es un instrumento valioso pero hay que des-neoliberalizarlo}

El género es en un instrumento valioso que nos sirve para denunciar la opresión de lo masculino hacia lo femenino. Se asocia lo masculino al hombre y lo femenino a la mujer. Para recuperar al género hay que descolonizarlo y des-neoliberalizarlo, y esto porque no hay otro concepto en la actualidad que haya mostrado mejor la situación y condición de las mujeres, como el género. Ojo, no la equidad de género, sólo el género.

El género tiene como valor político lo mismo que la clase, nunca va a haber equidad (igualdad) de clase porque las clases sociales se originan en la injusticia de la explotación de una sobre la otra: los burgueses sobre los proletarios. La lucha en este caso consiste en la superación de la clase como realidad histórica y la inauguración de una etapa de la humanidad donde no hayan explotados ni explotadores, es decir, que se terminen las clases sociales.

De modo análogo, nunca va a haber equidad de género, entendida como igualdad, porque el género masculino se construye a costa del género femenino, por lo que la lucha consiste en la superación del género. De lo que se trata es de trascender el género como producto histórico-cultural y dar paso a una nueva forma de criar y socializar a las wawas. Lo que se quiere desde el feminismo comunitario es ya no ser más ni femeninas ni masculinos. Se trata de acabar con las relaciones de poder construidas por el género.

El chacha-warmi (complementariedad hombre-mujer en aymara), no tiene el instrumento de la denuncia del género. Necesitamos esa 
denuncia para poder entender y develar las causas de las condiciones de opresión de las mujeres y cambiarlas. Veremos más adelante cómo el chacha-warmi es también patriarcal y colonial.

\section{Nuestro Plan de las Mujeres es un Plan que exige reflexión, acción y lucha}

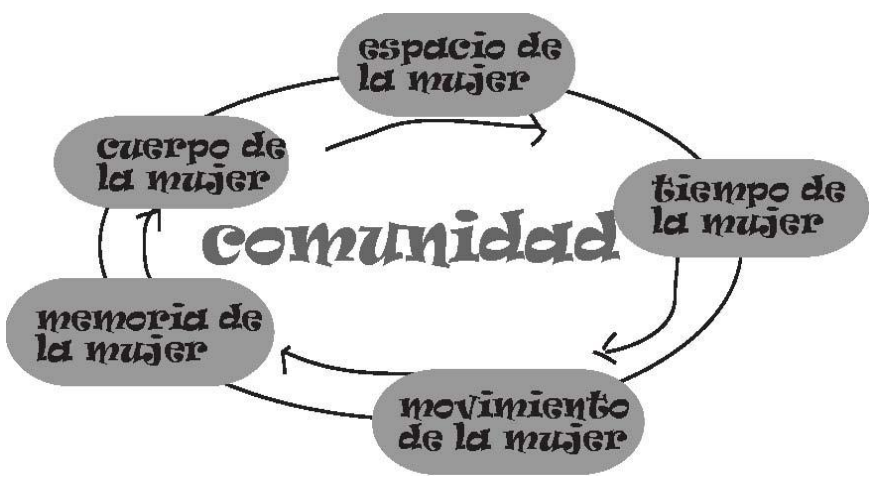

Es un Plan DINAMICO, siempre está proponiendo el movimiento de los campos de acción y lucha, tanto aisladamente, como en conjunto.

INTERRELACIONA dinámicamente los campos de acción, pero también INTERRELACIONA unas mujeres con otras, sean de la comunidad que sean, construyendo redes de reflexión, acción y lucha.

Es un Plan SUPERADOR de indicadores cuadrados autosuficientes, reduccionistas y separados entre sí, como eran los indicadores del neoliberalismo. En el neoliberalismo no se tenía un marco conceptual propio de las mujeres porque el marco conceptual era el neoliberalismo. Nuestro plan rompe con los cuadrados cerrados de los indicadores neoliberales y relaciona conceptos y categorías en los campos de acción y lucha por parte de nosotras las mujeres.

Es un Plan INCLUYENTE porque nace desde las organizaciones de base de las mujeres y propone que nos APROPIEMOS de él generando 


\section{REVISTA DE ESTUDIOS BOLIVIANOS}

indicadores dinámicos y locales, de manera que cada mujer, y las mujeres de cada organización y municipio, produzcan sus indicadores y los hagan cumplir.

\section{Esto está superado:}

\section{violencia}

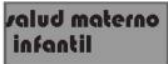

\section{participación} políticen

Pero el modelo que lo reemplaza, aunque pone la comunidad en relación a los indicadores - lo que supone una mejora del modelo anteriorsigue siendo más de lo mismo, porque no se trata ahora de relacionar cuadrados en comunidad:

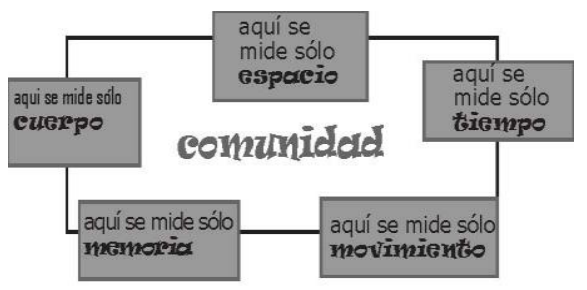

\section{se trated de una} comunidad que se muteve, o sed dinámica

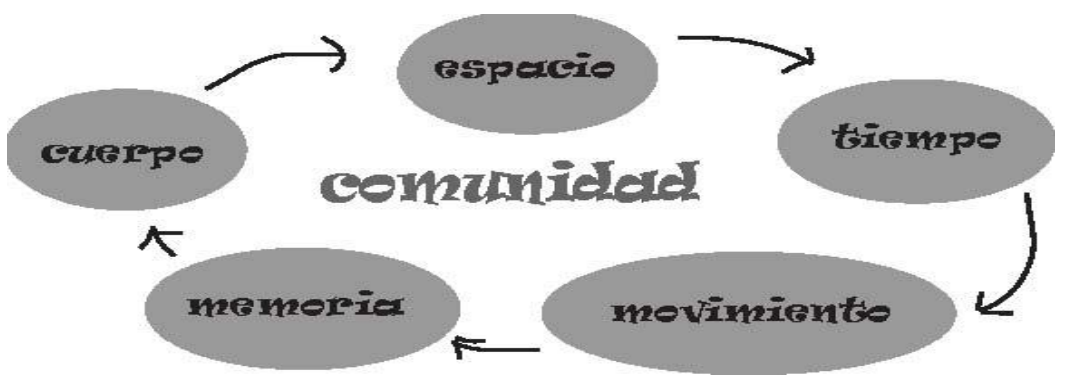




\section{Las mujeres somos la mitad de cada comunidad}

La comunidad está constituida por mujeres y hombres como dos mitades imprescindibles, complementarias, no jerárquicas, recíprocas y autónomas una de la otra. Las mujeres no somos la yapa de los hombres. Ser un par complementario no necesariamente significa una heterosexualidad obligatoria, porque no estamos hablando de "pareja" sino de "par", no estamos hablando de "familia" sino de "comunidad". Hablamos de la célula política de la sociedad, no hablamos de la célula reproductiva de la sociedad.

\section{¿Qué cosa es entonces la comunidad?}

Cuando decimos "comunidad", nos referimos a todas las comunidades de nuestra sociedad: urbanas, rurales, religiosas, deportivas, culturales, educativas, políticas, universitarias, de lucha, territoriales, agrícolas, barriales, generacionales, sexuales, comunidades de tiempo libre, comunidades de amistad, comunidades de afecto, etc. Es comprender que de todo y de todo grupo humano podemos hacer y construir comunidades.

Nuestro Plan de las Mujeres parte de la realidad de cada comunidad, de cada organización, asociación, colectivo, grupo, familia. Resulta entonces imprescindible, absolutamente necesario, que los varones participen de talleres donde reconozcan una primera realidad: que las mujeres somos la mitad de todo, que tenemos derechos y los vamos a hacer cumplir.

Los varones deben empezar a entender que el mundo es par. Somos dos diferentes que tenemos el mismo valor. No nos proponemos quitar nada a los hombres, sólo vamos a tomar lo nuestro, nuestra mitad en cada comunidad. Consideramos un gravísimo error negar el cuerpo y sexo de quienes formamos parte de los movimientos y organizaciones sociales. Son nuestros cuerpos de mujeres que desde siempre, en innumerables marchas y acciones, han hecho y construido la historia de nuestro país. Recuperamos pues el par complementario, pero no el par 


\section{REVISTA DE ESTUDIOS BOLIVIANOS}

machista del chacha-warmi, que en la realidad significa los hombres arriba y privilegiados y las mujeres abajo y explotadas. Al chacha-warmi hay que des-patriarcalizarlo, descolonizarlo y des-neoliberalizarlo para que nos sirva como instrumento revolucionario para construir una nueva sociedad. Replanteamos el par complementario en warmi-chacha, mujerhombre, warmi-k'ari, cuna-avá, que no es un simple cambio de lugar de las palabras, es la reconceptualización del par complementario desde las mujeres, porque las mujeres somos las que estamos subordinadas, y construir un equilibrio y armonía en la comunidad y en la sociedad viene a partir de las mujeres, ahora es el tiempo de las mujeres.

\section{Cuerpo de las mujeres}

Partimos de una concepción integral del cuerpo que comprende desde la biogenética hasta la energética, desde la afectividad, pasando por la sensibilidad, el erotismo, la sensualidad, hasta la creatividad. Con nuestros cuerpos que quieren comer bien, estar sanos, que gustan de las caricias y les duelen los golpes, con nuestros cuerpos que quieren tener tiempo para conocer y hacer teorías, queremos nombrar las cosas desde nosotras, con el sonido de nuestra propia voz. Queremos poner el cuerpo en hacer movimientos sociales y políticos que recojan nuestras propuestas y junten nuestros sueños y esperanzas de mujeres. El cuerpo nuestro nos plantea recuperar nuestras energías y nuestra salud, queremos estar sanas y no morirnos al tener nuestras hijas e hijos, queremos mirarnos al espejo y amar nuestras formas corporales, nuestros colores de piel y los colores de nuestros cabellos, porque estamos hartas de una estética colonial y racista, además cansadas del espectáculo frívolo de cuerpos que se exhiben, parte de la superficialidad y culto a la apariencia que cultivó el neoliberalismo.

\section{Espacio de las mujeres}

Comprendemos el espacio como un campo vital para que el cuerpo se desarrolle. El espacio es donde la vida se mueve y promueve. Nos referimos a la casa, la tierra, la escuela, la calle, entendidos como partes 


\section{REVISTA DE ESTUDIOS BOLIVIANOS}

del espacio privado y del espacio público. Por otro lado, están los lugares de producción y de sustento diario: el espacio de la comunidad con tu tierra común y el territorio, la fábrica, el taller o el barrio donde se hace la vida en las ciudades y, por supuesto, el espacio político y el espacio cultural. La lectura nuestra del espacio comprende dos sentidos, uno horizontal y otro vertical, como DOS ENVOLVENTES que tratan de abrazar e incluir todo lo que propicia la vida y que además nos dan las coordinadas respecto a dónde se localiza la comunidad y desde dónde estamos hablando con ella.

La envolvente vertical recoge tres lugares verticalmente ubicados: el Arriba, el Aquí y el Abajo. El Arriba es el espacio que está por encima de la comunidad. Este espacio tiene que ver con el espacio aéreo, el espacio de las comunicaciones, telecomunicaciones, y de la comunicación satelital. El Aquí es donde estamos y transitamos las personas de las comunidades. $Y$ finalmente el Abajo es el lugar donde descansan nuestras antepasadas, las semillas, las raíces, los recursos naturales como el gas y el petróleo, las aguas termales y el calor de la tierra, los minerales y los animales subterráneos y también la energía y savia que nos da la Pachamama.

La envolvente horizontal del espacio recoge la extensión de la tierra y el territorio de la comunidad hasta sus límites y fronteras. Es aquí donde va a tomar sentido la comunidad, las autonomías indígenas, municipales, departamentales, y el Estado Comunitario.

\section{Tiempo de las mujeres}

El tiempo es una condición para la vida, porque la vida para las personas no es atemporal. Al contrario, la vida tiene una temporalidad que se expresa en las formas que toma el cuerpo. El envejecimiento es una de sus manifestaciones, y no siempre significa algo negativo. La vida que corre gracias al movimiento de los actos conscientes es sentida y percibida como tiempo. Pero a la vez es también una medida muy útil para la percepción de nosotras las mujeres, que hemos venido al mundo a Vivir Bien, y no a pasar el tiempo de nuestra vida sufriendo y siendo infelices. La medida del tiempo nos confronta y conflictúa porque entendemos que no somos eternas y que un día moriremos. Por eso tomar conciencia de nuestros tiempos va abriendo espacios para 


\section{REVISTA DE ESTUDIOS BOLIVIANOS}

producir procesos de transformación en nuestra cotidianeidad y en nuestra propia historia.

La visión cíclica y fundida al espacio que suele manejarse en las comunidades tiene dos formas de aplicarse a la realidad concreta de los cuerpos en la comunidad: hay un tiempo importante que corresponde a los hombres, donde ellos son privilegiados. Otro es el tiempo de las mujeres, que viven un tiempo no-importante, y por tanto succionado por el de los hombres.

Estas concepciones patriarcales del tiempo han planteado que lo que ocupa al hombre, o sea el tiempo del hombre, es valioso. Haga lo que haga el hombre, difícilmente para la sociedad él está perdiendo su tiempo. Los tiempos de las mujeres, en cambio, son degradados, percibidos como no-tan-importantes. Por eso se les paga menos, y el trabajo doméstico de las amas de casa no es valorado ni pagado.

\section{Movimiento de las mujeres}

El movimiento es una de las propiedades de la vida que garantiza su subsistencia. Si algo está vivo se mueve. En este caso estamos hablando de construir organización y propuestas sociales. El movimiento nos permite construir un cuerpo social, un cuerpo común que lucha por vivir y Vivir Bien. El movimiento nos garantiza que los derechos conquistados no se conviertan en instituciones pesadas que ahoguen las utopías por las cuales luchamos. Son hilos que con tácticas y estrategias, paciencia, pasión y compromiso, las mujeres de la comunidad van tejiendo y enlazando.

Pero además, el movimiento lleva en su seno algo mucho más importante que lo define en el camino y que son los procesos que se dan en medio. Si cargamos nuestros procesos por ejemplo de racismos o de corrupción, pues el movimiento que logremos ha de tener estos elementos y se va a volver en contra de nosotras. Por supuesto que aquí está presente la visión de país que las mujeres tenemos y su relación a un todo mayor desde el cual identificarnos y actuar. Este todo tampoco es cerrado, con fronteras como murallas, es más bien un todo parcial para actuar ahora, un todo que nos permite actuar y medir el tamaño de nuestras acciones internacionales. El movimiento nos da la sensación de 


\section{REVISTA DE ESTUDIOS BOLIVIANOS}

estar vivas cuidando y proyectando la vida. El movimiento es el lugar de la reapropiación de los sueños.

\section{Memoria de las mujeres}

Entendemos esta categoría como las raíces de las cuales venimos, que son únicas, propias de aquí. Es toda esa fuerza y energía lo que construye nuestra identidad desde antes que nacemos. Es la memoria la que nos enlaza con las antepasadas. Es esa forma de la vida que se ha dado en estas tierras y que es irrepetible. Podemos parecernos a otras, pero hay cosas que son únicas. Es entonces la información, la calidad de la energía, la novedad de las experiencias de estas tierras lo que nos convierte en aportadoras de saberes al conjunto de la humanidad. Desde las mujeres, entendemos la memoria como ese correr, desde tiempos inmemoriales, detrás de utopías, probando las frustraciones y éxitos que constituyen la materia de las raíces de las cuales procedemos.

El concepto de "memoria larga" usado en el indigenismo nos remite acríticamente a la época prehispánica como a algo mejor, casi perfecto para las mujeres, pero destruido por la desgracia de la colonia. Esta "memoria larga" es interesada, pues si bien otorga orgullo y dignidad de ser personas pertenecientes a pueblos con culturas y logros como cualquier otro pueblo, es selectiva en el momento de no reconocer patriarcalismos, opresiones, autoritarismos e injusticias heredados y que, por supuesto, estaban presentes en las sociedades prehispánicas. Hay que des-patriarcalizar la memoria. La memoria nos permite recoger a las mujeres rebeldes de nuestras comunidades en sus resistencias antipatriarcales. Nos permite también reconocer a las que hoy todavía están en las comunidades, algunas de ellas ancianitas, y valorar sus aportes a nuestras luchas como mujeres.

\section{Ahora iremos al plano de lo concreto en las políticas públicas nacionales}

¿Cómo se va a trabajar interrelacionando los campos arriba expuestos? ¿Cómo nuestro cuerpo, nuestro espacio, nuestro tiempo, 


\section{REVISTA DE ESTUDIOS BOLIVIANOS}

nuestro movimiento y nuestra memoria van a funcionar orgánica y estratégicamente dentro de la comunidad? Para que esto suceda, vamos a hacer uso de toda nuestra creatividad. Aquí sirven todas las dinámicas de grupo, técnicas y juegos aprendidos en los talleres. Todo es válido para que con nuestra creatividad nos apropiemos de este Plan de las Mujeres para que de verdad sea nuestro.

Para empezar, es necesario reflexionar sobre el significado de todos los campos de acción, entender cuáles son los modelos de sociedad que están en pelea y cuál es nuestro aporte como mujeres en estos procesos de cambio. En el campo específico del tiempo, hay que decir que éste atraviesa todos los demás y está presente en todos y cada uno como condición para que puedan interrelacionarse.

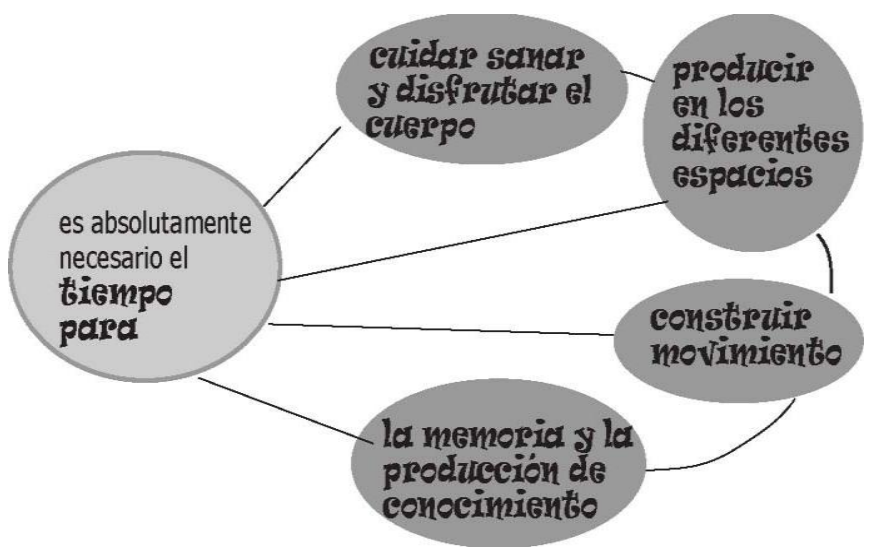

Una dinámica que ayuda a que se tome conciencia del Tiempo de las mujeres es la elaboración de papelógrafos, uno con el horario detallado de las actividades diarias de los hombres y otro con el de las mujeres, desde que se levantan hasta que se acuestan, para después compararlos.

Este ejercicio muestra que en el plano específico y local, mientras las mujeres trabajamos en el campo del Cuerpo, trabajamos simultáneamente por conquistar un Espacio (en el que podamos vivir sin 


\section{REVISTA DE ESTUDIOS BOLIVIANOS}

violencia y con libertad para ejercer nuestra sexualidad y nuestros placeres), recuperar un Tiempo nuestro, producir un Movimiento (capaz de obtener espacios de decisión y participación política) y restituir una Memoria de conocimiento sobre nuestros cuerpos de mujeres. De igual manera, mientras trabajamos en el campo específico del Espacio, luchamos simultáneamente por nuestros Cuerpos, nuestro Tiempo, nuestro Movimiento y nuestra Memoria. Descubrimos que en nuestras actividades todos los campos están vinculados y referidos a la comunidad a la que pertenecemos.

En lo que sigue, ofreceremos algunos ejemplos concretos de la ejecución de nuestro Plan en organizaciones sociales de mujeres. Trabajando el campo del Movimiento, analizamos la organización de mujeres de una villa en una de las ciudades de Bolivia y empezamos a aplicar el Plan de las Mujeres.

PRIMER PASO: en medio de tantos problemas de nuestro barrio, las mujeres nos damos cuenta que varias veces hemos apoyado a fórmulas políticas para que asuman la dirección de la Junta Vecinal, pero después han ignorado nuestras necesidades. Por ejemplo, queríamos utilizar la sede del barrio para hacer cursos de cocina y macramé y no quisieron darnos el local (que nosotras habíamos ayudamos a construir). A partir de este hecho comenzamos a organizarnos para presionar y que nos den el local, pero nada siempre. Entonces decidimos organizar nuestro grupo, le dimos un nombre y empezamos a reunirnos. Finalmente nuestro deseo es tomar la Junta Vecinal como mujeres y participar en las elecciones que también las propiciamos nosotras porque la Junta no quería salir.

SEGUNDO PASO: analizamos nuestros problemas inmediatos:

1. Las mujeres desconfiamos de nuestra capacidad y consideramos que eso de las elecciones es cosa de hombres.

2. Las compañeras no tienen tiempo para asistir a las reuniones y sus esposos ya están enojados porque sus mujeres no les atienden en casa y descuidan sus obligaciones.

TERCER PASO: es necesario trabajar con nuestras compañeras para que comprendan que las mujeres somos la mitad de la comunidad y que no solo tenemos derecho, sino OBLIGACIÓN de participar. Nosotras somos la mitad del cuerpo que es nuestro barrio o villa. 


\section{REVISTA DE ESTUDIOS BOLIVIANOS}

Para no cometer los mismos errores y ser lo mismo que la anterior Junta es necesario revisar lo que dijimos en la primera parte de este trabajo, donde establecemos que este es un proceso de cambios que pertenece al pueblo. También es necesario entender que las mujeres tenemos que ser más valientes, pues vamos a tener que luchar también en casa, porque hay un patriarcado popular que no nos quiere dejar surgir.

En el campo del Movimiento, las mujeres crean una fórmula política para participar en las elecciones. Realizan su campaña electoral con respeto pero con claridad y decisión en lo que desean para su villa y ganan por amplia mayoría. En el proceso se dan cuenta de que para votar o ser elegida, y luego para hacer trámites, las mujeres necesitamos un documento de identidad.

En el campo del Cuerpo, se trabaja por perder el miedo a expresar su opinión. Las mujeres hacen sus cursos de cocina dirigidos también a la salud. Valoran las opiniones de unas y otras, no hay mandonas ni racistas. Ya no tienen vergüenza de hacer cursos de sexualidad y conocen sus derechos sobre cuándo, cómo y con quién elegir ser mamá.

En el campo del Tiempo, las mujeres organizan talleres para sensibilizar a los hombres a compartir el trabajo doméstico y valorarlo. De esta manera se recupera el Tiempo de las mujeres y se reivindica el valor de sus ocupaciones.

En el campo del Espacio, logramos espacios de decisión y participación política. Las mujeres participan en la FEJUVE (Federación de Juntas Vecinales) y en la CONALJUVE (Consejo Nacional de Juntas Vecinales) y plantean los derechos de las mujeres en los barrios. Descubren que las mujeres de la fórmula no tenían los títulos de propiedad de sus casas a su nombre y proponen leyes para cambiar esa injusticia. Además, expulsan del barrio a los violadores.

En el campo de la Memoria, recuperamos la memoria de nuestra participación en la historia y construimos memoria documentada. Las mujeres se enteran de lo que son los POAs (Presupuesto Orgánico Anual), aprenden a pedir audiencias con el Consejo Municipal y el Alcalde, y preparan ordenanzas municipales favorables a ellas. Realizan encuentros con las mujeres más ancianas del barrio para conocer la historia de la villa y el papel que en ella tuvieron las mujeres. Todo esto se graba. También se organizan talleres para conocer los pensamientos de las mujeres, como por ejemplo el feminismo comunitario. Con nuestra Memoria y 
experiencia nutrimos el Movimiento, reproduciendo en la práctica el ciclo dinámico del Plan de las Mujeres.

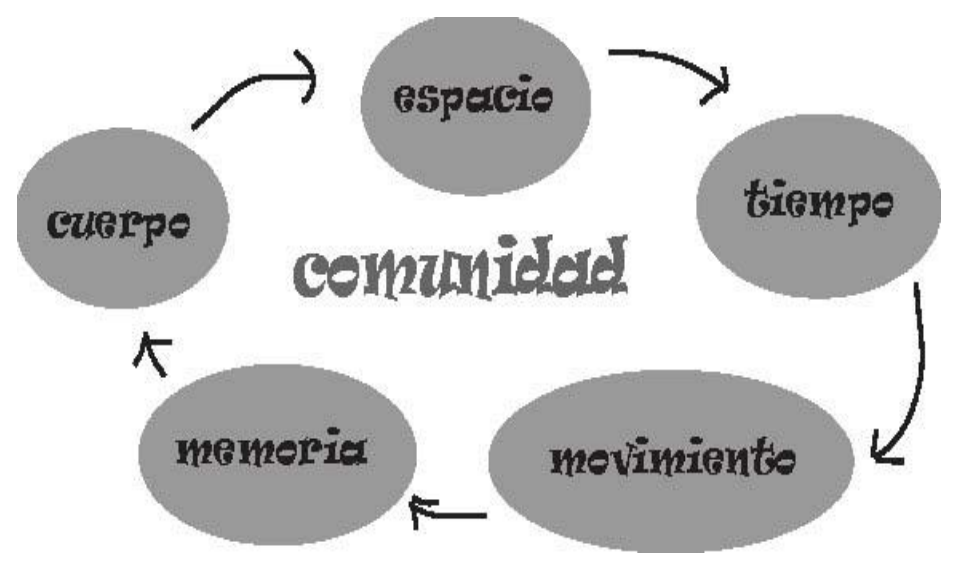

Otro ejemplo concreto de la ejecución de nuestro Plan de las Mujeres en organizaciones sociales de mujeres es el realizado en otra villa a partir del campo del Espacio.

PRIMER PASO: la comunidad tiene, desde hace algunos años, un proyecto de turismo comunitario. En medio de estos procesos de cambio, nos invitaron y pudimos participar de algunas reuniones. En ellas nos dimos cuenta que ahora existen créditos y préstamos especiales para actividades económicas de las mujeres. Hay muchas necesidades que tenemos nosotras, pero como el esposo es el que maneja el dinero y conoce el funcionamiento del turismo comunitario, a las mujeres en realidad no nos llegan los beneficios directamente y no tenemos dinero para disponer, siempre tenemos que estarle pidiendo al esposo. Por eso queremos ese proyecto productivo para las mujeres. 


\section{REVISTA DE ESTUDIOS BOLIVIANOS}

SEGUNDO PASO: analizamos nuestros problemas inmediatos:

1. Las mujeres desconfiamos de nuestra capacidad para manejar dinero de créditos y tampoco nos sentimos capaces de manejar el turismo comunitario desde las mujeres, consideramos que eso es cosa de hombres, mucho más si tiene que ver con turistas extranjeros.

2. Tenemos que reunirnos, pero las compañeras no tienen tiempo para las reuniones. Sus esposos están enojados porque sus mujeres no les atienden en casa y descuidan sus obligaciones.

TERCER PASO: es necesario trabajar con nuestras compañeras, ayudarles a entender que las mujeres somos la mitad de la comunidad y que no sólo tenemos derecho sino OBLIGACIÓN de participar. Nos ayuda repasar lo que dijimos anteriormente respecto a la comunidad, para que se entienda que sin nosotras la comunidad estaría, coja, manca, tuerta, sorda de un lado. Nosotras somos la mitad de ese cuerpo que es nuestra comunidad.

Para no cometer los mismos errores y ser lo mismo que la anterior Junta, es necesario tomar conciencia de lo que significa este proceso de cambio y por qué le pertenece al pueblo. También es necesario saber por qué las mujeres tenemos que ser más valientes, pues vamos a tener que luchar también en casa, donde un patriarcado popular no nos quiere dejar surgir. Todo esto está en las primeras páginas de este trabajo.

En el campo del Espacio, nos damos cuenta de que así como está, la comunidad es como si no fuera nuestra, pues sólo cuentan los nombres de los hombres. En la TCO (Territorio Comunitario de Origen) tampoco figuramos las mujeres como parte de la comunidad. Acordamos que la tierra es también nuestra y así debe figurar en los papeles de la comunidad. Nos proponemos sacar los créditos a nombre nuestro, porque son créditos para mujeres, y fundamos la Asociación de Turismo Comunitario, donde las mujeres somos al menos el $50 \%$.

En el campo del Cuerpo, nos damos cuenta de que estamos siempre cansadas y no tenemos fuerza. Por eso organizamos talleres para aprender a combinar mejor los alimentos. Vemos que la mayoría de las mujeres no tiene toda su dentadura y nos proponemos traer un dentista a la comunidad a través del proyecto productivo. Cuando sufrimos violencia o violaciones nos deprimimos y no nos da ganas de hacer nada, por eso no puede haber violencia hacia las mujeres, porque baja nuestro rendimiento y perturba nuestra dignidad. 


\section{REVISTA DE ESTUDIOS BOLIVIANOS}

En el campo del Tiempo, debido al trabajo doméstico, las mujeres no tenemos Tiempo. Por eso es imprescindible compartir este trabajo con los varones y monetarizar el trabajo doméstico de las amas de casa, para evitar la auto explotación de las mujeres y recuperar nuestro Tiempo.

En el campo del Movimiento, creamos una organización femenina de turismo para acceder a créditos. Además, participamos de la Asociación de Turismo Comunitario de mi pueblo originario, donde las mujeres somos al menos el $50 \%$ de la directiva. Ya en la directiva, presentamos nuestras propias propuestas para las autonomías indígenas y departamentales. En resolución de la Asamblea de la Comunidad, decidimos que para las reuniones se llama a hombre y mujer por su nombre, y que las casas son de hombre y mujer.

En el campo de la Memoria, organizamos el festival de las abuelas para que ellas nos muestren lugares y costumbres olvidadas por nosotras. Registramos todo en video. Después, organizamos la capacitación en turismo comunitario con apoyo del Vice-ministerio de Turismo. Nuevamente, en este caso, reproducimos en la práctica y desde la comunidad, el ciclo dinámico del Plan de las Mujeres.

\section{Logramos Espacios de decisión y participación política} Trabajamos por nuestros Cuerpos Recuperamos el Tiempo de las mujeres Creamos un Movimiento social de mujeres

Recuperamos la Memoria y la construimos desde las escuelas y centros educativos.

\section{(cc) $\mathrm{EY}-\mathrm{NC}-\mathrm{ND}$}

This work is licensed under a Creative Commons AttributionNoncommercial-No Derivative Works 3.0 United States License. 